\title{
Role of the Dorsal Anterior Cingulate Cortex in Relational Memory Formation: A Deep Brain Activity Index Study
}

\author{
Ariko Araki ${ }^{*}$, Emiko Imai ${ }^{1}$, Yoshitada Katagiri ${ }^{1,2}$ \\ ${ }^{1}$ Department of Rehabilitation Science, Kobe University Graduate School of Health Science, Kobe, Hyogo, Japan \\ ${ }^{2}$ Advanced ICT Research Institute, National Institute of Information and Communication Technology, Koganei, Tokyo, Japan \\ Email: *a.araki@stu.kobe-u.ac.jp
}

How to cite this paper: Araki, A., Imai, E. and Katagiri, Y. (2018) Role of the Dorsal Anterior Cingulate Cortex in Relational Memory Formation: A Deep Brain Activity Index Study. Journal of Behavioral and Brain Science, 8, 269-293.

https://doi.org/10.4236/jbbs.2018.85017

Received: January 12, 2018

Accepted: May 13, 2018

Published: May 16, 2018

Copyright ( $) 2018$ by authors and Scientific Research Publishing Inc. This work is licensed under the Creative Commons Attribution International License (CC BY 4.0).

http://creativecommons.org/licenses/by/4.0/

\begin{abstract}
The dorsal area of the anterior cingulate cortex (ACC) constructs the salience network associated with the anterior insular cortex. Conventional brain imaging studies, such as functional magnetic resonance imaging (fMRI), have demonstrated that relational memory formation occurs in the ACC. However, how such memory is encoded and retrieved remains unknown due to limited time resolution of conventional fMRI. This study aimed to investigate temporal dynamics of the dorsal ACC (AACC) during word-pair tasks based on a newly developed event-related deep brain activity (ER-DBA) method using occipital electroencephalogram (EEG) signal powers. The method assesses dACC activity at a temporal resolution of approximately $0.3 \mathrm{~s}$ beyond the conventional resolution limit. We found that transient deactivation of dACC during the presentation of the second word of each pair was essential for encoding success regardless of whether the words were related or unrelated. We also found that memory accuracy was not affected by the intervention of inter-trials until the recall trial. Taken together, these findings suggest that dACC deactivation for encoding success is accompanied with short-term potentiation essential for durability of memory. We further found that false memory formation associated with the presentation of word pairs was occasionally committed. In such cases, dACC exhibited a similar transient deactivation although false memory commission was independent of related or unrelated conditions. Our findings suggest that encoding and retrieval of associates are paralleled and that simultaneous production of associates seems to be an essential strategy for successful relational memory formation. The study was limited to the assessment of dACC activity and did not account for other regional brain activities or receptor regulation related to short-term potentiation. We detected fast behavior of dACC during relational memory formation
\end{abstract}


using the novel ER-DBA method. Such temporal dynamics will be important for eliciting underlying mechanisms of memory dysfunctions.

\section{Keywords}

Relational Memory, Word-Pair Task, Event-Related Deep Brain Activity, Dorsal Anterior Cingulate Cortex, Temporal Dynamics

\section{Introduction}

Traditionally, memory functions were believed to be regulated by the hippocampus and the medial temporal lobe (MTL) [1] [2] [3] [4]. Previous studies have investigated the mechanisms of memory formation and found that the MTL was deactivated during encoding and activated during recall [5] [6].

The deactivation of MTL during encoding is counter-intuitive as the hippocampus plays a primary role in durable memory formation. The hippocampus may, therefore, be dissociable from the network related to MTL [7] including the default-mode network (DMN), comprising of the ventromedial prefrontal cortex, the posterior cingulate cortex, the retrosplenial cortex, the inferior parietal lobule, and the hippocampus. The hippocampus can remain activated during durable memory formation independently of the behaviors of DMN. Despite such dissociation, encoding and recall processes are thought to be spatially [8] and temporally [9] integrated [3]. While considering durable memory formation in daily life, memory and attention are not dissociable brain functions [10] [11] [12], and the ventral parietal cortex regulates these two brain processes [13] [14] [15]. Recent studies have highlighted the importance of the ventromedial prefrontal cortex in causing cognitive and memory dysfunctions in dementia [16] [17] [18].

Clinical evidence demonstrating that semantic-dementia patients maintain intact episodic memory despite severe atrophy of the hippocampus contradicts theoretical frameworks relying on MTL functions [19] [20]. Such clinical evidence has triggered the emergence of theories relying on large-scale networks associated with cognitive control rather than on MTL functions. These large-scale networks include the salience network (SN), central executive network (CEN) [21] [22], and the Papez circuit [23]. Novel theoretical frameworks are needed to bridge the boundaries between memory and cognitive processing [12] [24].

A candidate novel framework could be based on the anterior cingulate cortex (ACC) that has been hypothesized to contribute to the consolidation of recent and remote memories associated with information transferred from the hippocampus [25] [26] [27] in animal models [28]. The ACC (particularly the dorsal anterior cingulate cortex; $\mathrm{dACC}$ ) is related to attention control and forms SN with the anterior insular cortex and regulates CEN and DMN while responding to external events [29]-[34]. Memory and attention may therefore overlap in 
dACC.

We hypothesized that $\mathrm{dACC}$ is directly related to durable memory formation and that its temporal activity reflects encoding and recall processes during memory formation, assuming that the two memory processes are temporally dissociable. We tested this hypothesis by investigating dACC functions during durable memory formation. The activity of dACC was assessed with a noninvasive technique using occipital electroencephalogram (EEG) alpha-2 $(10-13 \mathrm{~Hz})$ power defined as a deep brain activity (DBA) index [35] [36]. Past studies have focused on identifying regions associated with occipital alpha power [37] [38] [39] and found that regions are dependent on the frequency range of the power fluctuation [40]. A higher component $(\geq 0.04 \mathrm{~Hz})$ primarily reflects activity of dACC while a slow component $(\leq 0.04 \mathrm{~Hz})$ reflects activity of the region surrounding the upper brainstem and involving the monoaminergic neural systems [40]. We extended this technique to develop an event-related paradigm with trial-by-trial measurements. We used temporal traces with typical time windows of only a few seconds to evaluate dACC activity. Temporal resolution was estimated at below a few hundreds of milliseconds thereby accounting for the frequency range of alpha-2. The temporal resolution was suitable for our study despite the temporal limitations imposed by the conventional event-related fMRI paradigm [41].

We used word-pair tasks in our experiment. Word-pair tasks are associated with relational memory [3] that is consolidated in CEN [42] [43] whereas relational processing is originally undertaken in the hippocampus [44] [45]. Dynamic behaviors of dACC could, therefore, reflect relational memory formation during word-pair tasks.

\section{Materials and Methods}

\subsection{Subjects}

Subjects were recruited from Kobe University. Twelve healthy volunteers (six males and six females) from the ages of 27 to 44 (mean $=32$; standard deviation, $\mathrm{SD}=5.3)$ with no historical records of hearing impairment and psychiatric diseases participated in the study. They provided written informed consent in accordance with the protocol approved by the ethical committee of Kobe University Graduate School of Health Sciences (No. 529). All subjects were therapists with similar higher-education levels.

\subsection{Word-Pair Tasks}

We adopted two word-pair lists for the word-pair tasks. The lists included 30 commonly used and semantically related (i.e., lion versus tiger) or unrelated (i.e., snake versus bread) Japanese nouns. A total of 60 pairs were used in this study. The nouns were extracted from the standard verbal paired-associate learning test (S-PA) with permission of the Japan Society for Higher Brain Dysfunction [46]. 
The experimental procedure consisted of two sessions using the two word-pair lists (Figure 1(A)). Each session included 30 trials that were composed of encoding, retention, and recall phases. In accordance with the protocol of the S-PA, the session using the related word-pair list was conducted first and the session using the unrelated word-pair list was conducted second. The subjects knew in advance which list was to be used for the coming session. The order of the word pairs did not change among subjects. The memory test was replicated with the two word-pair lists. Each word-pair was auditorily presented to subjects in a trial with an average duration of $6.5 \mathrm{~s}$. The duration of each trial was randomized in the range of 6 - $7 \mathrm{~s}$ and included substantial inter-trial intervals of a few seconds. The duration of the encoding phase was approximately 3.5 min. Following the encoding phase, the recall phase started at an interval of 3 min. The sequence of events for the recall phase was similar to that of the encoding phase. An equivalent duration of $3.5 \mathrm{~min}$ was assigned for promoting the recall phase. Each session was completed in $10 \mathrm{~min}$. As the intermittent interval between the two sessions was several minutes, the total experimental period per subject was approximately $25 \mathrm{~min}$. The sequence of events in a trial is depicted in Figure 1(B) for the encoding and recall phases.

(A)

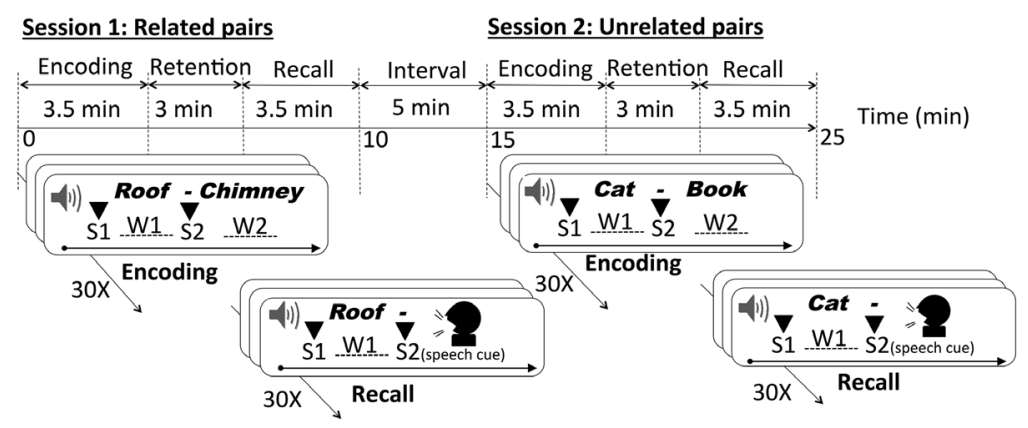

(B)

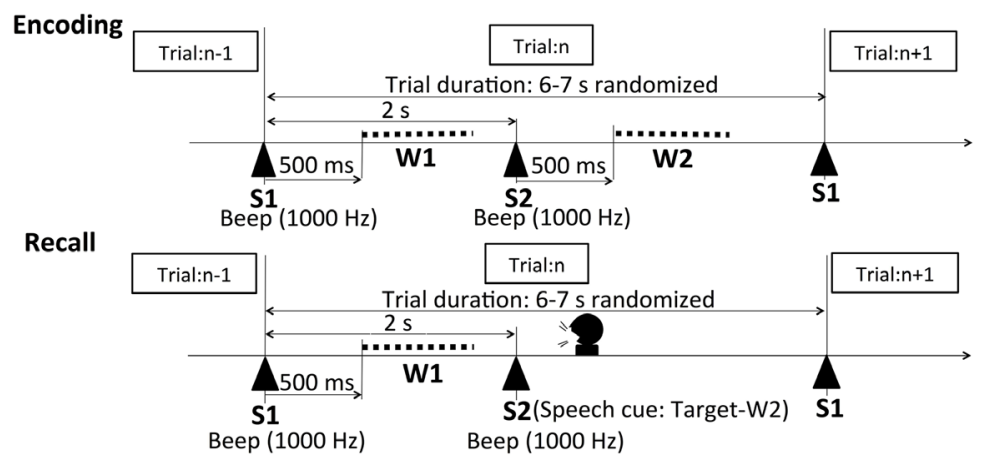

Figure 1. Experimental design. (A) Schematic overview of the setup consisting of two sessions using word-pair lists that included related or unrelated pairs. The presentation order was rearranged in the recall phase. (B) One trial of the encoding and recall phases, comprising beep sound signals (S1 and S2). S1 corresponds to an event marker of both encoding and recall phases for analyses by arithmetic mean. S2 is used as a cue for speech during the tasks. Pairs of words (W1-W2) are automatically presented by a PC after the S1 and S2 signals. 
In the encoding phase, the paired words were auditorily presented to the subjects by using digital data recorded with a voice recorder by a native speaker. The word pairs W1 and W2 were sequentially presented after the event markers S1 and S2, respectively. The two event markers were separated by $2 \mathrm{~s}$. The interval between the onset of the word presentation and the event marker was approximately $500 \mathrm{~ms}$. At the event markers, beep sounds with frequencies of 1000 $\mathrm{Hz}$ were presented to the subjects as prior stimulation to maintain attention. The word pairs (W1-W2) were presented only once. In the encoding phase, the subjects were asked to remember word pairs to induce a delayed recall.

After the encoding session, a recall test started within a few minutes. Such prompt recall test was promoted to distinct encoding failure from exponential decrease of memory accuracy. In the recall phase, the subjects were given the first word (W1) and then asked to orally state the target word (W2) upon presentation of the speech cue (S2). The interval between S1 and S2 was 2 s. The subjects were prevented from ignoring the speech cue in silence (i.e., they were asked to say "Forget" if they had forgotten the target word). The subjects' responses were recorded on a voice recorder for behavioral analyses. The order of the word pairs varied among the task phases but did not change among subjects.

\subsection{EEG Recordings}

Scalp EEG signals were recorded from $\mathrm{Ag} / \mathrm{AgCl}$ electrodes aligned in accordance with the international $10-20$ system. The recording was conducted under the eye-open condition using a digital EEG recorder with a sampling frequency of $512 \mathrm{~Hz}$ and 24-bit analogue-to-digital converters grounded at the AFZ site of the 10 - 10 system. The montage data were generated with references from the mastoid electrodes.

\subsection{Performance Analyses}

Performance on the word-pair tests was assessed using the subjects' responses measured on the voice recorder in the recall phase. The performance assessment was repeated for each trial and all trial responses were grouped into three response groups: high memory accuracy (HA), medium memory accuracy (MA), and low memory accuracy (LA). HA responses were defined as accurately remembering the target word, whereas LA responses were defined as forgetting the target word. HA responses were identified by verifying whether the generated words corresponded with the targets. LA responses were detected by self-assessment by stating "Forget". MA was a discordant response defined as stating a word that differed from the target word. MA was not numerically assessed due to the lack of clear measures for quantitatively evaluating semantic distance between the correct (target) and incorrect (falsely generated) words. Semantic similarity between the false word and target was assessed with multiple experimenters to control for inter-judge reliability. When the false word was similar to the first word of the pair (W1), the false trial was labeled as MA (W1). 
We similarly defined MA (W2) for the incorrect word similar to W2. Inadequate responses in the recall phase such as missing speech cues, regarded as commission error (CE), were excluded from performance analyses.

\subsection{EEG Data Analyses}

In our word-pair tasks, words were sequentially presented according to the trial-by-trial design that presented word pairs separated by only $2 \mathrm{~s}$ (Figure 1). Direct comparison across trials was needed for investigating the dynamic behaviors of dACC specific to encoding success or failure. However, conventional event-related fMRI paradigms were unsuitable for this purpose due to a limited time resolution $>1 \mathrm{~s}$.

We developed an ER-DBA method with a time resolution of approximately $300 \mathrm{~ms}$ to investigate task-oriented activities of deep brain structures for cognitive studies, including dACC and upper brainstem [47]. We adopted this method for our study and details are described below. The activities of the deep brain structures including dACC and upper brainstem can be numerically evaluated from the DBA index defined as the average of the occipital EEG alpha-2 $(10-13 \mathrm{~Hz})$ powers at the $\mathrm{O} 1$ and $\mathrm{O} 2$ sites calculated every $31.25 \mathrm{~ms}$ with a $2 \mathrm{~s}$ epoch based on a conventional Fast Fourier Transform algorithm. According to a previous study [40], these two components are dissociable by the critical fluctuation frequency of $0.04 \mathrm{~Hz}$. The higher frequency component represents the activity of dACC whereas the lower frequency component represents that of the upper brainstem, primarily the monoaminergic neural systems. The ER-DBA method refers to a conventional event-related paradigm that uses event markers for producing event-specific neural responses by arithmetic averaging. A detailed procedure is illustrated in Figure 2. The event marker S1 represented the onset of each trial and was automatically recorded along the EEG signals to extract trial data. A typical time window of $4 \mathrm{~s}$ corresponded to the segments used in this study and provided a cut-off frequency of $0.25 \mathrm{~Hz}$, much higher than the critical frequency. The ER-DBA traces represented at this time window depicted the temporal dynamics of dACC. The event marker S1 was subsequently relabeled according to task performance. Segmental data were extracted with respect to each relabeled code to produce performance-dependent ER-DBA traces. Since the relative timings of word presentations were fixed across all trials, we assessed event-specific brain responses on the reference of the relabeled onset markers.

\subsection{Statistical Analysis}

\subsubsection{Significant Memory Accuracy and Error Incidence}

Behavioral responses were grouped into performance categories for all subjects. To investigate the effects of lexical similarity on relational memory formation, paired t-tests were performed to detect differences in memory accuracy and CE incidence between related and unrelated pairs. To determine whether the false $\mathrm{W} 1$ and $\mathrm{W} 2$ responses were similar, paired t-tests were performed between the 


\section{Superposed waves \\ and ER-DBA traces with respect to maker codes}
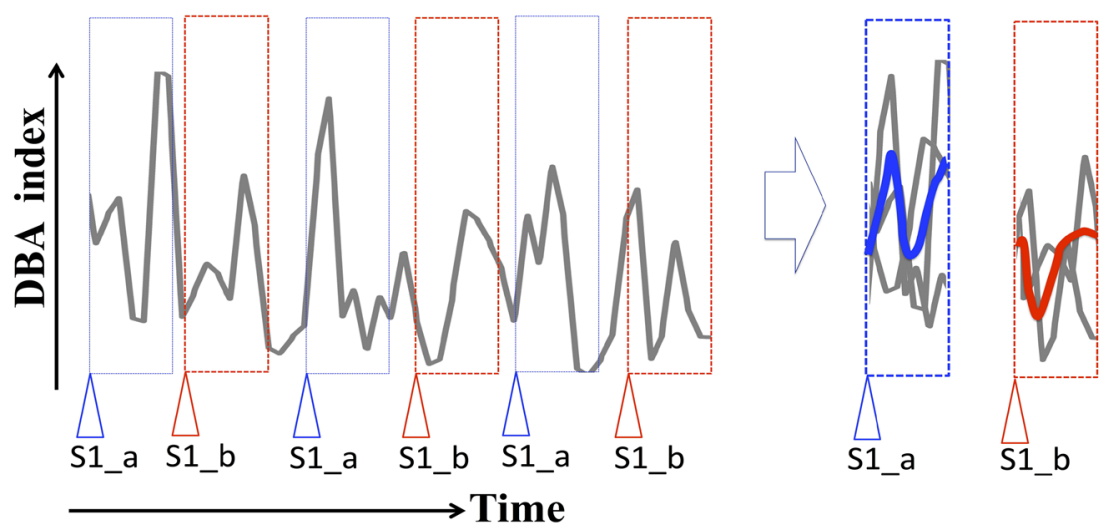

Figure 2. Time-series signals of the deep brain activity (DBA) index with two event markers coded by S1_a and S1_b (left panel) and event-related DBA (ER-DBA) traces extracted from signals corresponding to each event marker (right panel). The markers corresponded with performance categorized to high memory accuracy, low memory accuracy, and medium memory accuracy as described in Section 2.4 Performance Analyses. The time-series signals were cut in a limited time window of approximately $4 \mathrm{~s}$, including header intervals of $200 \mathrm{~ms}$ prior to the markers for baseline correction. ER-DBA traces were calculated with an arithmetic mean with respect to each marker.

two MA groups. We further investigated how the presented words affected memory accuracy. Statistical analyses using paired t-tests were performed between the MA (W1) and MA (W2) groups.

\subsubsection{Evaluation of Statistical Reliability for the ER-DBA Traces}

The baseline of the ER-DBA was used to assess whether dACC was activated or deactivated. Statistical significance for the assessment was numerically evaluated with the standard error of the mean (SE). The statistical evaluation was conducted for every trace accompanied by a shaded area corresponding to $1.96 \mathrm{SE}$ to show significant activation or deactivation at a significance level of 0.05 . Deactivations regarded as dips in ER-DBA traces were characterized by depth and duration. Depth was assessed by the bottom of the traces. Width was numerically evaluated using the full width at half maximum (FWHM).

\section{Results}

\subsection{Behavioral Performance Data}

Memory performance was evaluated using recorded speech data and represented by task scores across subjects (Table 1). The performance assessment was performed with category codes as HA, LA, MA, MA (W1), MA (W2), and CE, according to the criteria as described in Materials and Methods.

Numerical analyses on behavioral performance data revealed that related word pairs had much higher HA response incidence than unrelated pairs ( $p<$ 0.01; Figure 3(A)). Further, LA (forgotten) responses exhibited much higher in- 
cidence for unrelated pairs than those for related pairs $(p<0.01$; Figure 3(B)). On the other hand, we also found a total discordant response feature depicted by MA-All responses with much higher $(p<0.05)$ incidence for unrelated pairs than those for related pairs (Figure $3(C)$ ). We further examined details of the discordant response feature with MA (W1) and MA (W2) responses. A significant difference $(p<0.01)$ was detected between MA (W1) and MA (W2) for related pairs (Figure 3(D)). Although no significant difference $(p=0.065)$ was detected for unrelated pairs, the corresponding effect size was not negligible $($ Cohens'd $=0.90)$ (Figure 3(E)). Such differences in MA responses were due to the much decreased incidence of MA (W2) responses for related pairs.

Table 1. Behavioral performance data of 12 subjects when performing word-pair tasks consisting of (a) related and (b) unrelated word-pair sessions. (a) Related word pair; (b) Unrelated word pair.

(a)

\begin{tabular}{|c|c|c|c|c|c|c|c|c|c|c|c|c|}
\hline \multicolumn{3}{|c|}{ Subjects } & \multicolumn{5}{|c|}{ Response $^{\mathrm{a}}(\%)$} & \multicolumn{5}{|c|}{ Trial $^{\mathrm{b}}$} \\
\hline \multirow[b]{2}{*}{ ID } & \multirow[b]{2}{*}{ Age } & \multirow[b]{2}{*}{ Sex } & \multirow[b]{2}{*}{ HA } & \multirow[b]{2}{*}{ LA } & \multicolumn{2}{|c|}{ MA } & \multirow[b]{2}{*}{$\mathrm{CE}$} & \multirow[b]{2}{*}{ HA } & \multirow[b]{2}{*}{ LA } & \multicolumn{2}{|c|}{ MA } & \multirow[b]{2}{*}{$\mathrm{CE}$} \\
\hline & & & & & (W1) & (W2) & & & & (W1) & (W2) & \\
\hline 1 & 38 & $\mathrm{~F}$ & $\begin{array}{c}23 \\
(76.7)\end{array}$ & $\begin{array}{c}6 \\
(20.0)\end{array}$ & $\begin{array}{c}1 \\
(3.3)\end{array}$ & 0 & 0 & $\begin{array}{c}1-5,7-18, \\
21-23,25-27\end{array}$ & $\begin{array}{l}6,19,20 \\
24,29,30\end{array}$ & 28 & - & - \\
\hline 2 & 39 & $\mathrm{M}$ & $\begin{array}{c}24 \\
(80.0)\end{array}$ & $\begin{array}{c}3 \\
(10.0)\end{array}$ & $\begin{array}{c}1 \\
(3.3)\end{array}$ & $\begin{array}{c}1 \\
(3.3)\end{array}$ & 1 & $\begin{array}{l}1-3,5,6,8-16 \\
18,19,22,24-30\end{array}$ & $4,17,21$ & 7 & 20 & 23 \\
\hline 3 & 29 & $\mathrm{~F}$ & $\begin{array}{c}18 \\
(60.0)\end{array}$ & $\begin{array}{c}9 \\
(30.0)\end{array}$ & $\begin{array}{c}2 \\
(6.7)\end{array}$ & $\begin{array}{c}1 \\
(3.3)\end{array}$ & 0 & $\begin{array}{c}1-7,10-13, \\
16-18,23-25,27\end{array}$ & $\begin{array}{c}8,14, \\
19-22,28-30\end{array}$ & 9,26 & 15 & - \\
\hline 4 & 29 & $\mathrm{M}$ & $\begin{array}{c}24 \\
(80.0)\end{array}$ & $\begin{array}{c}4 \\
(13.3)\end{array}$ & $\begin{array}{c}1 \\
(3.3)\end{array}$ & 0 & 1 & $\begin{array}{c}1-3,5-15, \\
17-20,22,24-28\end{array}$ & $\begin{array}{l}16,21 \\
29,30\end{array}$ & 4 & - & 23 \\
\hline 5 & 30 & $\mathrm{~F}$ & $\begin{array}{c}22 \\
(73.3)\end{array}$ & $\begin{array}{c}6 \\
(20.0)\end{array}$ & $\begin{array}{c}2 \\
(6.7)\end{array}$ & 0 & 0 & $\begin{array}{c}1-6,8,10-13,15 \\
18-21,23,25-27,29,30\end{array}$ & $\begin{array}{l}7,14,16, \\
22,24,28\end{array}$ & 9,17 & - & - \\
\hline 6 & 30 & $\mathrm{M}$ & $\begin{array}{c}27 \\
(90.0)\end{array}$ & $\begin{array}{c}2 \\
(6.7)\end{array}$ & $\begin{array}{c}1 \\
(3.3)\end{array}$ & 0 & 0 & $1-20,22-28$ & 29,30 & 21 & - & - \\
\hline 7 & 28 & $\mathrm{~F}$ & $\begin{array}{c}21 \\
(70.0)\end{array}$ & $\begin{array}{c}6 \\
(20.0)\end{array}$ & $\begin{array}{c}2 \\
(6.7)\end{array}$ & $\begin{array}{c}1 \\
(3.3)\end{array}$ & 0 & $\begin{array}{c}1-8,11-18 \\
20,23,24,26,27\end{array}$ & $\begin{array}{l}9,10,19 \\
21,29,30\end{array}$ & 22,28 & 25 & - \\
\hline 8 & 27 & $\mathrm{~F}$ & $\begin{array}{c}25 \\
(83.3)\end{array}$ & $\begin{array}{c}2 \\
(6.7)\end{array}$ & $\begin{array}{c}2 \\
(6.7)\end{array}$ & $\begin{array}{c}1 \\
(3.3)\end{array}$ & 0 & $\begin{array}{l}1-7,9,10,12-15 \\
17-20,22-28,30\end{array}$ & 21,29 & 11,16 & 8 & - \\
\hline 9 & 44 & $\mathrm{~F}$ & $\begin{array}{c}23 \\
(76.7)\end{array}$ & $\begin{array}{c}7 \\
(23.3)\end{array}$ & 0 & 0 & 0 & $\begin{array}{c}1-8,10,12-21 \\
23,24,26,27\end{array}$ & $\begin{array}{c}9,11,22, \\
25,28-30\end{array}$ & - & - & - \\
\hline 10 & 28 & M & $\begin{array}{c}28 \\
(93.3)\end{array}$ & $\begin{array}{c}2 \\
(6.7)\end{array}$ & 0 & 0 & 0 & $1,3-28,30$ & 2,29 & - & - & - \\
\hline 11 & 32 & $\mathrm{M}$ & $\begin{array}{c}25 \\
(83.3)\end{array}$ & $\begin{array}{c}4 \\
(13.3)\end{array}$ & $\begin{array}{c}1 \\
(3.3)\end{array}$ & 0 & 0 & $1,3-13,15-26,30$ & $\begin{array}{l}2,14 \\
28,29\end{array}$ & 27 & - & - \\
\hline 12 & 33 & $\mathrm{M}$ & $\begin{array}{c}27 \\
(90.0)\end{array}$ & $\begin{array}{c}2 \\
(6.7)\end{array}$ & 0 & 0 & 1 & $1-22,24-27,30$ & 28,29 & - & - & 23 \\
\hline
\end{tabular}


(b)

\begin{tabular}{|c|c|c|c|c|c|c|c|c|c|c|c|c|}
\hline \multicolumn{3}{|c|}{ Subjects } & \multicolumn{5}{|c|}{ Response $^{\mathrm{a}}(\%)$} & \multicolumn{5}{|c|}{ Trial $^{\mathrm{b}}$} \\
\hline \multirow[b]{2}{*}{ ID } & \multirow[b]{2}{*}{ Age } & \multirow{2}{*}{ Sex } & \multirow{2}{*}{ HA } & \multirow{2}{*}{ LA } & \multicolumn{2}{|c|}{ MA } & \multirow[b]{2}{*}{$\mathrm{CE}$} & \multirow{2}{*}{ HA } & \multirow{2}{*}{ LA } & \multicolumn{2}{|c|}{ MA } & \multirow{2}{*}{$\mathrm{CE}$} \\
\hline & & & & & (W1) & (W2) & & & & (W1) & (W2) & \\
\hline 1 & 38 & $\mathrm{~F}$ & $\begin{array}{c}18 \\
(60.0)\end{array}$ & $\begin{array}{c}10 \\
(33.0)\end{array}$ & $\begin{array}{c}1 \\
(3.3)\end{array}$ & $\begin{array}{c}1 \\
(3.3)\end{array}$ & 0 & $\begin{array}{c}1-5,7,9,11,12, \\
16-18,21-25,30\end{array}$ & $\begin{array}{c}6,8,14,15 \\
19,20,26-29\end{array}$ & 13 & 10 & - \\
\hline 2 & 39 & $\mathrm{M}$ & $\begin{array}{c}19 \\
(63.3)\end{array}$ & $\begin{array}{c}8 \\
(26.7)\end{array}$ & $\begin{array}{c}2 \\
(6.7)\end{array}$ & $\begin{array}{c}1 \\
(3.3)\end{array}$ & 0 & $\begin{array}{l}1-3,5,9-12,14,16,17 \\
19-21,23,24,27,29,30\end{array}$ & $\begin{array}{c}4,6-8,15 \\
18,22,28\end{array}$ & 13,25 & 26 & - \\
\hline 3 & 29 & $\mathrm{~F}$ & $\begin{array}{c}16 \\
(53.3)\end{array}$ & $\begin{array}{c}13 \\
(43.3)\end{array}$ & 0 & $\begin{array}{c}1 \\
(3.3)\end{array}$ & 0 & $\begin{array}{c}2,5,7,8,10,11, \\
14-18,21,22,27,29,30\end{array}$ & $\begin{array}{c}1,4,6,9,12,13 \\
19,20,23-26,28\end{array}$ & - & 3 & - \\
\hline 4 & 29 & M & $\begin{array}{c}8 \\
(26.7)\end{array}$ & $\begin{array}{c}17 \\
(56.7)\end{array}$ & $\begin{array}{c}1 \\
(3.3)\end{array}$ & $\begin{array}{c}4 \\
(13.3)\end{array}$ & 0 & $1,6,7,11,16,18,20,29$ & $\begin{array}{c}2-5,8-10,13,15,19 \\
21,22,24,26-28,30\end{array}$ & 25 & $\begin{array}{l}12,14 \\
17,23\end{array}$ & - \\
\hline 5 & 30 & $\mathrm{~F}$ & $\begin{array}{c}18 \\
(60.0)\end{array}$ & $\begin{array}{c}7 \\
(23.3)\end{array}$ & 0 & $\begin{array}{c}4 \\
(13.3)\end{array}$ & 1 & $\begin{array}{l}2,3,5,7,10-15,17 \\
18,22,25,26,28-30\end{array}$ & $1,4,6,9,16,23,24$ & - & $\begin{array}{l}8,20 \\
21,27\end{array}$ & 19 \\
\hline 6 & 30 & $\mathrm{M}$ & $\begin{array}{c}13 \\
(43.3)\end{array}$ & $\begin{array}{c}14 \\
(46.7)\end{array}$ & 0 & $\begin{array}{c}3 \\
(10.0)\end{array}$ & 0 & $\begin{array}{c}1,2,7,9,11,17,19 \\
21-24,29,30\end{array}$ & $\begin{array}{c}3-6,8,10,12-15 \\
18,20,27,28\end{array}$ & - & $\begin{array}{c}16 \\
25,26\end{array}$ & - \\
\hline 7 & 28 & $\mathrm{~F}$ & $\begin{array}{c}6 \\
(20.0)\end{array}$ & $\begin{array}{c}19 \\
(63.3)\end{array}$ & $\begin{array}{c}1 \\
(3.3)\end{array}$ & $\begin{array}{c}4 \\
(13.3)\end{array}$ & 0 & $2,5,7,13,17,26$ & $\begin{array}{c}1,3,4,6,8-12,14-16 \\
19-22,25,27,30\end{array}$ & 18 & $\begin{array}{l}23,24 \\
28,29\end{array}$ & - \\
\hline 8 & 27 & $\mathrm{~F}$ & $\begin{array}{c}8 \\
(26.7)\end{array}$ & $\begin{array}{c}22 \\
(73.3)\end{array}$ & 0 & 0 & 0 & $\begin{array}{c}4,7,16,21, \\
23,24,25,26\end{array}$ & $\begin{array}{c}1-3,5,6,8-15 \\
17-20,22,27-30\end{array}$ & - & - & - \\
\hline 9 & 44 & $\mathrm{~F}$ & $\begin{array}{c}8 \\
(26.7)\end{array}$ & $\begin{array}{c}19 \\
(63.3)\end{array}$ & 0 & $\begin{array}{c}2 \\
(6.7)\end{array}$ & 1 & $\begin{array}{c}1,4,7,17 \\
20,21,24,30\end{array}$ & $\begin{array}{c}2,5,6,8,9,11-16 \\
18,19,23,25-29\end{array}$ & - & 3,22 & 10 \\
\hline 10 & 28 & $\mathrm{M}$ & $\begin{array}{c}13 \\
(43.3)\end{array}$ & $\begin{array}{c}16 \\
(53.3)\end{array}$ & $\begin{array}{c}1 \\
(3.3)\end{array}$ & 0 & 0 & $\begin{array}{l}1,3,7,8,11,12,14, \\
15,17,21,25,26,30\end{array}$ & $\begin{array}{c}2,4-6,9,10,16 \\
18-20,22-24,27-29\end{array}$ & 13 & - & - \\
\hline 11 & 32 & $\mathrm{M}$ & $\begin{array}{c}12 \\
(40.0)\end{array}$ & $\begin{array}{c}14 \\
(46.7)\end{array}$ & $\begin{array}{c}2 \\
(6.7)\end{array}$ & $\begin{array}{c}1 \\
(3.3)\end{array}$ & 1 & $\begin{array}{c}1,2,5-7,15, \\
17,21,22,25,29,30\end{array}$ & $\begin{array}{c}3,4,8-11,14,16, \\
19,20,23,24,26,28\end{array}$ & 13,18 & 12 & 27 \\
\hline 12 & 33 & $\mathrm{M}$ & $\begin{array}{c}11 \\
(36.7)\end{array}$ & $\begin{array}{c}17 \\
(56.7)\end{array}$ & $\begin{array}{c}1 \\
(3.3)\end{array}$ & $\begin{array}{c}1 \\
(3.3)\end{array}$ & 0 & $\begin{array}{c}2,7,11,14,18, \\
21,23,25,26,29,30\end{array}$ & $\begin{array}{c}1,3-6,8-10,12,15 \\
16,19,20,22,24,27,28\end{array}$ & 13 & 17 & - \\
\hline
\end{tabular}

${ }^{a}$ Numbers for each response represent the number of times that response was given by the subject out of a total of 30 trials per session. HA: high memory accuracy response; LA: low memory accuracy response; MA: medium memory accuracy response stating false words; MA (W1/W2): medium-accuracy response stating false words similar to either W1 or W2; CE: commission error. ${ }^{b}$ Numbers indicate the $\mathrm{n}^{\text {th }}$ word-pair that categorized HA, LA, or MA for the related session and HA, LA, MA (W1), or MA (W2) for the unrelated session.

False words that differed from the target word were regarded as discordant responses denoted by MA (medium memory accuracy). We obtained 17 MA responses for the related condition and 31 for the unrelated condition among the 12 subjects. Table 2 lists the results of the semantic analysis along with the false words and corresponding word pairs for W1 and W2.

To ascertain the robustness of the initial encoded memory, we examined the HA response (remembered) rate versus the number of inter-trials $(\Delta \mathrm{N})$ involved in the retention period until later recall (Figure 4(A)). The results showed that 


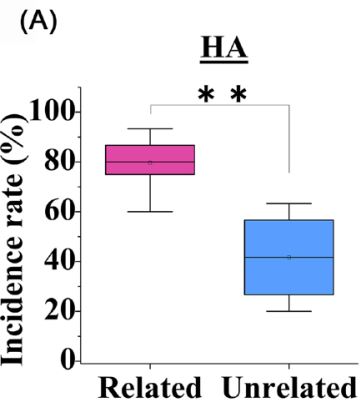

(D)

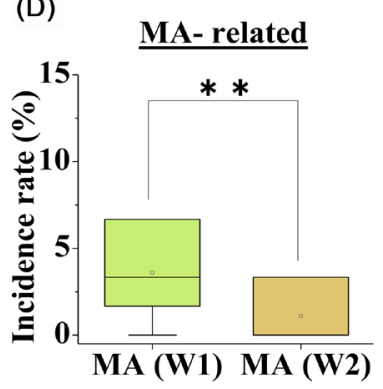

(B)

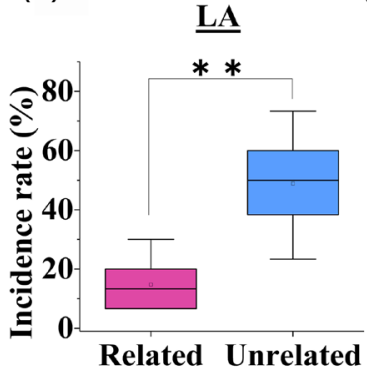

(C)

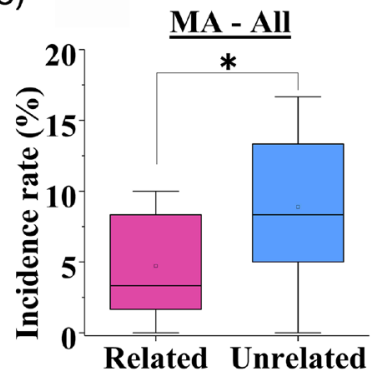

(E)

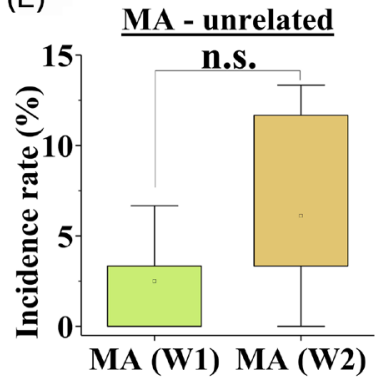

Figure 3. Numerical analyses on behavioral responses across 12 subjects. (A) Differences in HA response (remembered) incidence between the related and unrelated pairs (Cohen's $\mathrm{d}=3.1, p=10^{-5}$, power $=1.0$ ). (B) Differences in LA response (forgotten) incidence between the related and unrelated pairs (Cohen's $\mathrm{d}=2.8, p=10^{-5}$, power $=1.0$ ). (C) Differences in MA response (discordant) incidence between the related and unrelated pairs (Cohen's $d=0.87, p=0.049$, power $=0.52$ ). Differences in discordant response incidence between MA (W1) responses stating false words associated with W1 and MA (W2) responses stating those associated with W2 for (D) related pairs (Cohen's $\mathrm{d}=1.1, p$ $=0.0015$, power $=0.97)$ and $(\mathrm{E})$ unrelated pairs (Cohen's $\mathrm{d}=0.90, p=0.065$, power $=$ 0.46). HA, high memory accuracy; LA, low memory accuracy; MA, medium memory accuracy; MA (W1/W2), MA commission with discordant words close to W1/W2; ${ }^{*}, p<$ $0.05 ;{ }^{* *}, p<0.01 ;$ n.s., no significance.

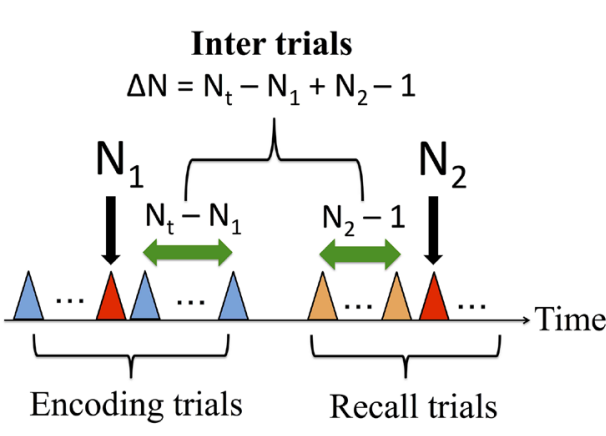

(A)

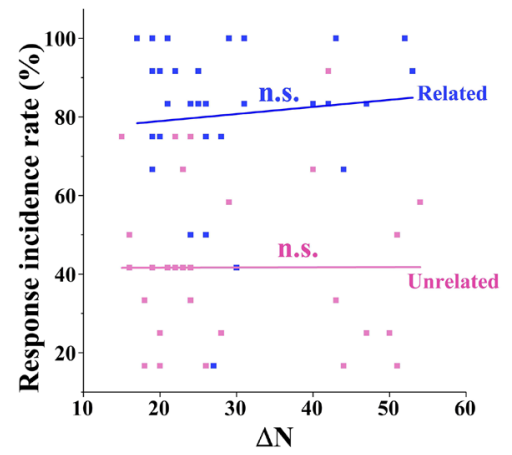

(B)

Figure 4. Initial encoded memory stability against the intervention of inter-trials. (A) Definition of inter-trial number $\Delta N$ defined as the number of trials included in the retention period until the recall trial using $N_{t} N_{l}$, and $N_{2}$ as the total trial number, the presentation orders in the encoding phase, and the presentation order in the recall phase, respectively. (B) No significant correlation between HA response (remembered) rate and inter-trial number for the related $(\mathrm{r}=0.095, p=0.61)$ and unrelated $(\mathrm{r}=0.0021, p=0.99)$ word pairs. 
Table 2. Whole word-list generated as false words with corresponding word pairs. The number embedded in false words is the frequency of commission errors with the same false word. The right-hand column represents the assessment of semantic similarity with W1 or W2.

\begin{tabular}{|c|c|c|c|}
\hline \multicolumn{4}{|c|}{ Related word pair-MA } \\
\hline W1 & W2 & False answer & Assessment \\
\hline Ju-do & Sumo & Kendo (2) & W1 \\
\hline Railroad & Station & Train (1) & W1 \\
\hline Rice & Miso-soup & Dish (1) & W1 \\
\hline Shower & Bath & Towel (1) & W1 \\
\hline Yukata & Paper fan & Belt (1) & W1 \\
\hline Soy-sauce & Sauce & Salt (1) & W1 \\
\hline Chest & Closet & Drawer (1) & W1 \\
\hline Marathon & Relay race & Relay (1) & W1 \\
\hline Boots & Umbrella & Rain (1) & W1 \\
\hline Salt & Sugar & Pepper (1) & W1 \\
\hline Sea & Mountain & Ship (1) & W1 \\
\hline Sea & Mountain & River (1) & W1 \\
\hline Sun & Moon & Cloud (1) & W2 \\
\hline Kendo & Karate & Ju-do (1) & W2 \\
\hline Hospital & Pharmacy & Medicine (1) & W2 \\
\hline Lion & Tiger & Zebra (1) & W2 \\
\hline \multicolumn{4}{|c|}{ Unrelated word pair-MA } \\
\hline W1 & W2 & False answer & Assessment \\
\hline Lip & Can & Skin (5) & W1 \\
\hline Pimple & Rubber band & Cream (2) & W1 \\
\hline Teacher & Dragonfly & Music (1) & W1 \\
\hline Teacher & Dragonfly & Mike (1) & W1 \\
\hline Fountain & Saw & Cutter knife (2) & W2 \\
\hline Trumpet & Turtle & Frog (2) & W2 \\
\hline Glass & Newspaper & Paper (2) & W2 \\
\hline Ham & Sword & Cutter knife (1) & W2 \\
\hline Ham & Sword & Saw (1) & W2 \\
\hline Seaweed & Earthworm & Mouse (1) & W2 \\
\hline Seaweed & Earthworm & Snake (1) & W2 \\
\hline Hairdryer & Thunder & Cutter knife (1) & $\mathrm{W} 2$ \\
\hline Goldfish & Ladder & Cutter knife (1) & W2 \\
\hline Sardine & Sunflower & Sun (1) & W2 \\
\hline Gargle & Rainbow & $\operatorname{Sun}(1)$ & W2 \\
\hline Beach & Stew & Soup (1) & W2 \\
\hline Hammer & Cherry Blossoms & Fountain (1) & W2 \\
\hline Pimple & Rubber band & Rubber (1) & W2 \\
\hline Police car & Tuna & Whale (1) & W2 \\
\hline Clown & Fan & Hairdryer (1) & W2 \\
\hline Frog & Scoop & Cutting board (1) & W2 \\
\hline Violin & Cutter knife & Saw (1) & W2 \\
\hline Autumn & Pearl & Heart (1) & W2 \\
\hline
\end{tabular}


no significant correlation was detected for the related $(r=0.095, p=0.61)$ and unrelated ( $r=0.0021, p=0.99)$ pairs. This result indicated that HA (remembered) responses reflected the initial encoding success, without any intervention of inter-trials from encoding until later recall (Figure 4(B)).

\subsection{Event-Related (ER) DBA Results}

Figure 5 shows event-related (ER) DBA traces for encoding with respect to behavioral performances classified as HA, LA, and MA by integrating all trials among the 12 subjects. Each trace had a 95\% confidence interval represented by a shaded area to detect the portions of the traces signaling significant deactivation or activation. Using this technique, we found that HA responses, including 196 samples in total for related pairs, provided significant $(p<0.05)$ DBA
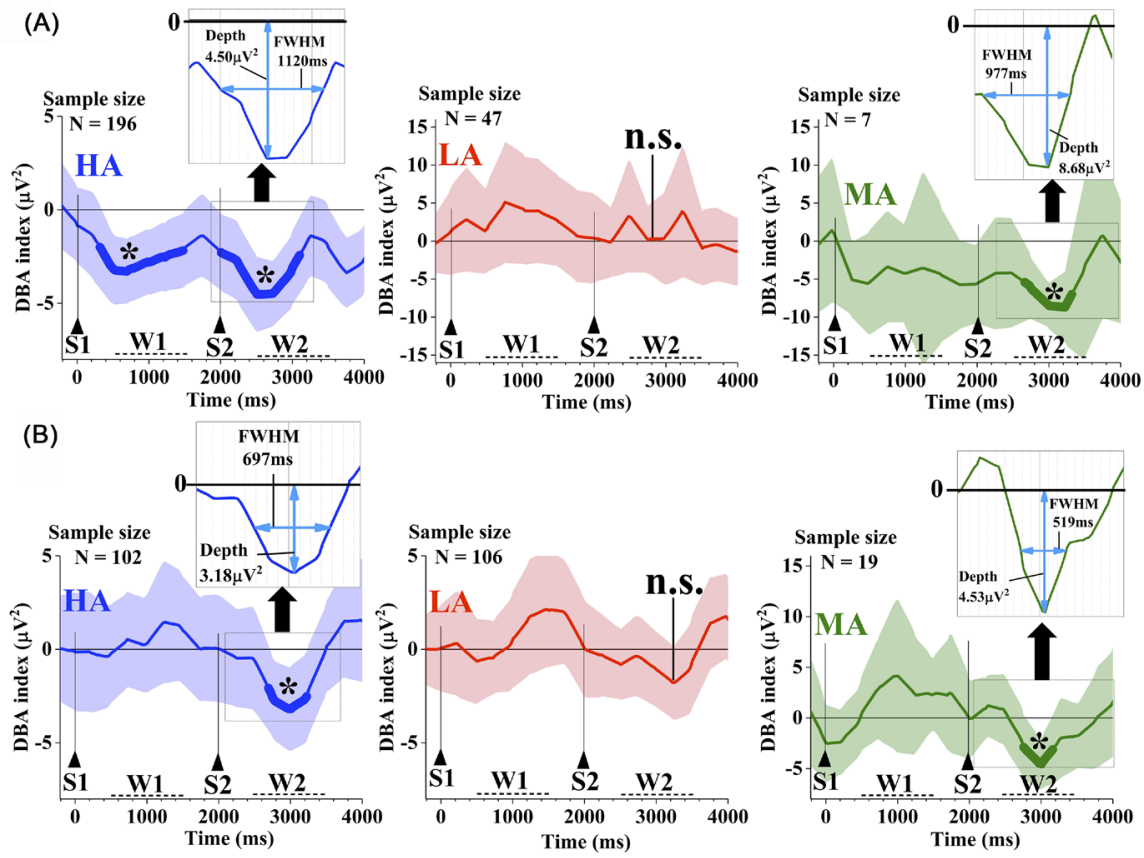

Figure 5. Performance-dependent event-related deep brain activity (ER-DBA) traces during encoding for (A) related and (B) unrelated pairs. Criteria for classifying behavioral performance to HA (high memory accuracy), LA (low memory accuracy), and MA (medium memory accuracy) are described in the Materials and Methods. Shaded areas for each trace show 95\% confidence intervals $(p<0.05)$ corresponding to 1.96 standard error of the mean (SE) assuming a normal distribution. Thicker portions on the lines represent significant deactivation $(p<0.05)$. Numerical features of the deactivation dips were analyzed with depth and width as shown in each inset panel. Deactivation was regarded as a dip for HA (related; during W1 presentation) $(\mathrm{N}=196, \mathrm{~d}=0.39, p<0.05$, power $=0.97)$, HA (related; during W2 presentation) $(\mathrm{N}=196, \mathrm{~d}=0.52, p<0.05$, power $=0.99)$, HA (unrelated) $(\mathrm{N}=102, \mathrm{~d}=0.39, p<0.05$, power $=0.78)$, MA (related) $(\mathrm{N}=7$, $\mathrm{d}=2.0, p<0.05$, power $=0.88)$, and MA (unrelated) $(\mathrm{N}=19, \mathrm{~d}=1.2, p<0.05$, power $=$ 0.94). The other responses, incuding LA responses for related and unrelated pairs, exhibited no deactivation with any significance (related: $p=0.91$; unrelated: $p=0.065$ ). $\mathrm{N}$, sample size; $\mathrm{d}$, effect size (Cohen's d); ${ }^{*}, p<0.05$; HA, high memory accuracy; LA, low memory accuracy; MA, medium memory accuracy. 
deactivation during the first (W1) and second (W2) word presentations. In contrast, we found that HA responses including 102 samples provided significant ( $p$ $<0.05)$ DBA deactivation only during the second word presentation. MA responses, regarded as encoding success in spite of imperfect memory formation accompanied with discordant responses stating incorrect words, were found to be significantly $(p<0.05)$ deactivated for the related and unrelated pairs, whereas they provided small sample sizes of $\mathrm{N}=7$ and 19, respectively. LA responses did not show any significant deactivation or activation on the ER-DBA traces.

As shown in each inset panels, these dips were characterized by depth and duration, while the depth was assessed by the bottom of the traces during the W2 presentation. The width was numerically evaluated by using FWHM.

Figure 6 shows the ER-DBA traces for recall with respect to behavioral performance as classified for encoding. The traces were also locked to the trial onset signal (S1). The probe word (W1) was presented posterior to the onset signal in
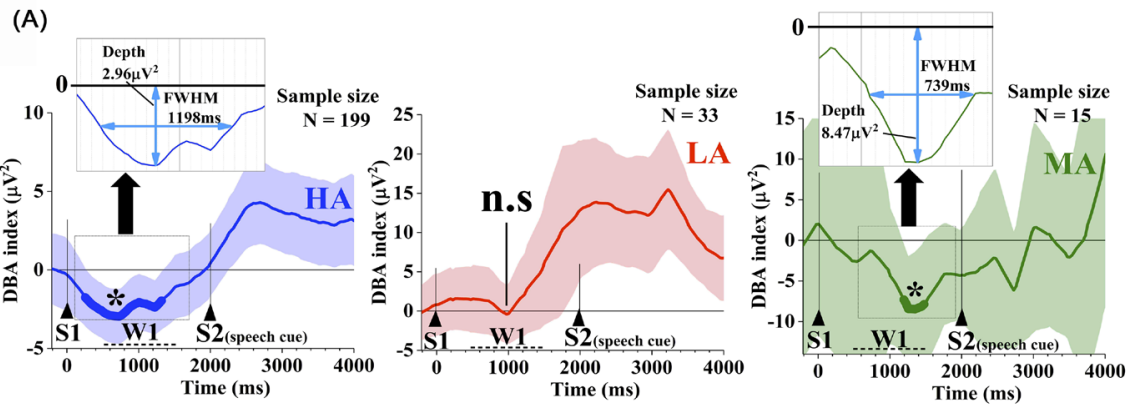

(B)
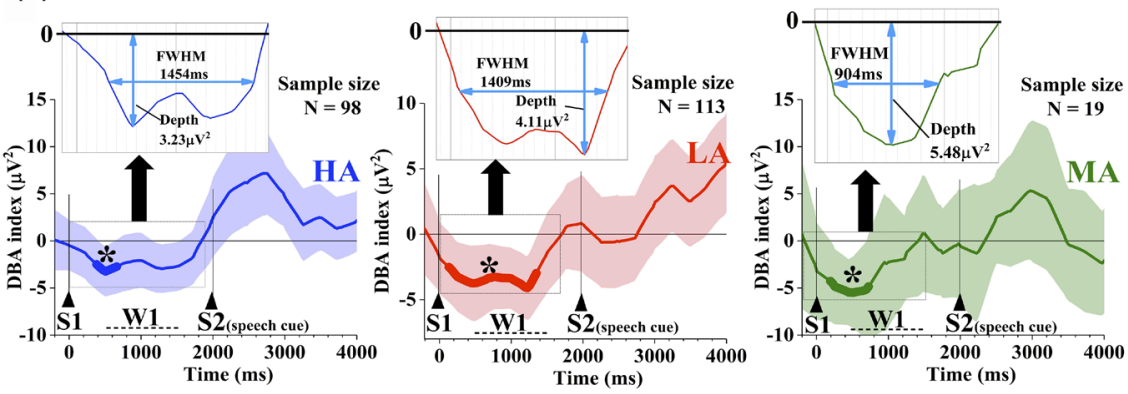

Figure 6. Performance-independent event-related deep brain activity (ER-DBA) traces during recall for (A) related and (B) unrelated word pairs. Criteria for classifying behavioral performance to HA, LA, and MA are described in the Materials and Methods. Shaded areas for each trace show 95\% confidence intervals $(p<0.05)$ corresponding to 1.96 standard error of the mean (SE) assuming a normal distribution. Thicker portions on the lines represent significant deactivation $(p<0.05)$. Numerical features of the deactivation dips were analyzed with depth and width as shown in each inset panel. The significant deactivation regarded as a dip was obtained during probe word (W1) presentation for HA (related) $(\mathrm{N}=199, \mathrm{~d}=0.36, p<0.05$, power $=0.95)$, HA (unrelated) $(\mathrm{N}=98$, $\mathrm{d}=0.34, p<0.05$, power $=0.70)$, MA (related) $(\mathrm{N}=15, \mathrm{~d}=0.99, p<0.05$, power $=0.71)$, and MA (unrelated) $(\mathrm{N}=19, \mathrm{~d}=1.1, p<0.05$, power $=0.89)$. LA (unrelated) also provided significant deactivation $(\mathrm{N}=113, \mathrm{~d}=0.45, p<0.05$, power $=0.92)$. LA (related) showed no significant $(p=0.37)$ deactivation during probe presentation. $\mathrm{N}$, sample size; $\mathrm{d}$, effect size (Cohen's $\mathrm{d}$ ); ${ }^{*}, p<0.05$; HA, high memory accuracy; LA, low memory accuracy; MA, medium memory accuracy. 
an interval of around $500 \mathrm{~ms}$. The speech cue (S2) was delay by $2 \mathrm{~s}$ from the onset signal (S1). When the speech cue arrived, the subjects randomly spoke, but they provided right answers or the forgetting sign. The traces exhibited significant deactivation during probe word (W1) presentation for almost all performances including HA (related: $p<0.05$ ), HA (unrelated: $p<0.05$ ), MA (related: $p<0.05$ ), and MA (unrelated: $p<0.05$ ). LA (unrelated) also provided significant deactivation $(p<0.05)$, whereas only LA (related) showed no significance.

We further characterized deactivations (dips) on ER-DBA traces for HA and MA responses during encoding and recall phases. We found that the widths and depths were narrower and deeper, respectively, for MA than those for HA responses. As shown in Figure 7(A), the differences helped distinguish the HA and MA groups in terms of width vs depth plots by an appropriate boundary, which is represented by a dotted line. Such differences indicate that MA responses provide stronger and quicker deactivations than HA responses, whereas the statisitical evidence was weak $(p<0.05$ for depth and no significance was found in terms of width) (Figure 7(B) and Figure 7(C)).

\section{Discussion}

This study aimed to investigate the temporal dynamics of dACC during word-pair tasks. Using a novel ER-DBA method with high temporal resolution compared with conventional imaging methods, we identified mechanisms underpinning relational memory formation.

\subsection{Critical Behavior of dACC during Encoding for Memory Formation}

From the ER-DBA results in the encoding phase (Figure 5), we found that correct

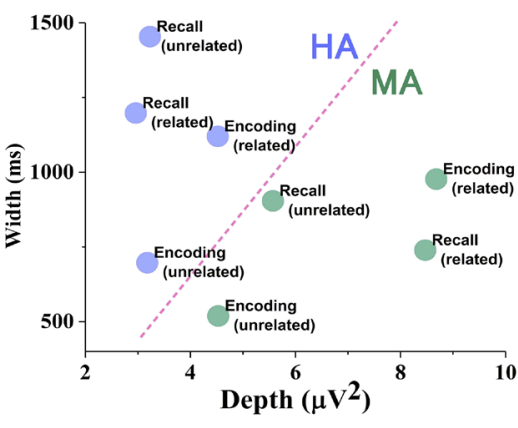

(A)

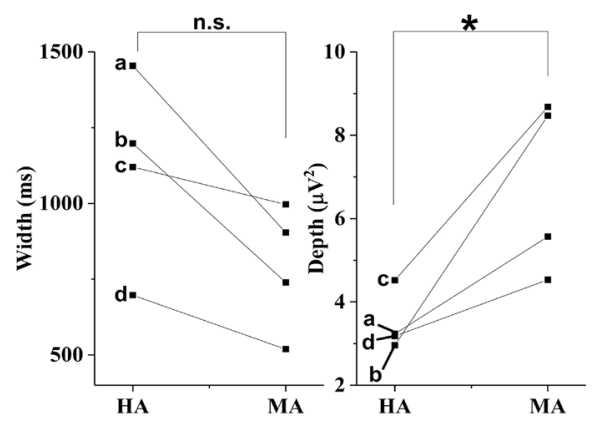

(B)

(C)
Figure 7. Numerical analyses of dips on ER-DBA traces for HA and MA responses. (A) Widths versus depths for the deactivation of event-related deep brain activity (ER-DBA) traces in encoding and recall phases. Definition of depth and width is schematically represented in Figure 5 and Figure 6. The differences in the (B) width and (C) depth of the dip on ER-DBA traces between HA and MA responses. The differences in depth were significant $(\mathrm{d}=2.1, \mathrm{p}<0.05$, power $=0.66)$ whereas the width was not significant $(\mathrm{d}=$ $1.2, p=0.052$, power $=0.56$ ). a, Recall (unrelated); b, Recall (related); c, Encoding (related); d, Encoding (unrelated); ${ }^{\star}, \mathrm{p}<0.05$. 
responses marked as HA were associated with significant deactivation of dACC during presentation of the second word (W2) as target in late recall independently of the task condition (related or unrelated). In contrast, incorrect responses marked as LA did not show any significant deactivation. Responses stating false words and marked as MA showed dACC deactivation patterns similar to those of correct responses. These results suggest that successful relational memory formation including imperfect memory (MA) is predicted by deactivation of dACC in the encoding phase. We also found from the behavior performance results that memory was stable at least in the experimental period, avoiding any influence of inter-trial intervention from encoding until recall (Figure 4(B)). We hypothesize that deactivation of dACC is associated with synaptic plasticity essential for non-volatile memory storage.

Previous studies have revealed the role of the ACC in forming immediate, recent, and remote memories. For recent and remote memories, the $\mathrm{N}$-methyl-D-aspartate receptor (NMDAR) is considered to be essential for memory durability accompanied with synaptic plasticity because activated NMDARs contribute to memory stabilization by increasing the number of alpha-amino-3-hydroxy-5-methyl-4-isoxazole-propionate (AMPA) receptors [48] [49]. Pharmacological studies have revealed that ACC deactivation is essential for NMDAR-activated synaptic plasticity regarded as long-term potentiation [50] [51] [52] [53] [54]. Further memory consolidation is promoted by c-Fos expression in the ACC that reversely behaves with the NMDAR-activated null mutation of the $\mathrm{Ca}^{2+} / \mathrm{calmodulin}$-dependent protein kinase II induced by ACC activation [55].

Synaptic plasticity can occur even in the formation of short-term memory and the ACC is associated with this process regarded as short-term potentiation (STP) [56] [57]. Similar to NMDAR-activated plasticity, STP requires ACC deactivation during encoding to produce giant action potentials from the hyper-polarized state [58] [59]. The effects of STP on the stabilization of working memory (a function of the CEN regulated by dACC as a node of the SN) have also been demonstrated in animal models [60]. NMDARs play a key role in the formation of all types of memories and dACC deactivation can be considered as an electrophysiological marker of NMDAR activation. The features of the cortical behaviors specific to STP mentioned by previous studies support our hypothesis. Importantly, deactivation occurs in a limited time window $(<1 s)$ at FWHM for encoding (Figure 5), which indicates that dACC is deactivated on demand and synchronized with external stimuli. We consider that such synchronous dACC behavior is a function of SN.

\subsection{Paralleled Encoding and Retrieving Processes for Relational Memory Formation}

From the results associated with MA responses stating false words semantically associated with the target in the late recall session (Table 2), we hypothesized that the false words had originated from simultaneous retrieval during encoding. 
We found that AACC was deactivated during the presentation of the second word (W2) in the encoding phase (Figure 5). In a previous study [47] we reported similar dACC deactivation during the generation of verbs semantically similar to presented nouns and concluded that dACC deactivation promotes target-oriented cognitive control by limiting null impulses incoming to dACC. Taken together, these findings suggest that parallel encoding and retrieval of associates are promoted for relational memory formation.

Various types of parallel information processes in memory formation have been reported, including integration of dissociable functional processes based on compartmentalization [61] [62] [63] and parallel phonological encoding and semantic processing [64]. Parallel information processes also include parallel activation for encoding and late retrieval in different regions associated with the hippocampus and the prefrontal cortex [8]. However, parallelisms of encoding and recall have remained unclear. We considered how the parallel recall of associates affects encoding success in a word network model (Figure 8). The model depicts a word cluster comprising associates for each word that are allocated according to semantic distance [65]. The area of overlap bridges the two words of interest and a relationship is formed between them. As semantic distance decreases, the bridge more tightly binds the words and corresponding relational memory becomes more robust. Parallel retrieval of associates during encoding is therefore beneficial for relational memory formation. Such dACC manners can be attributed to cost-effective strategy of human brain including economic decision-making paradigms [66] [67].

Importantly, it was suggested that a series of cognitive processes associated with relational memory formation was completed in a short time window corresponding to the narrow FWHM duration of dACC deactivation of $<1 \mathrm{~s}$. Our recent study found that dACC deactivation was correlated with upper brainstem activity associated with the monoaminergic neural systems at the ventral tegmental area [47]. Taken together, these findings provide an insight that cognitive processing associated with relational memory formation can be supported by

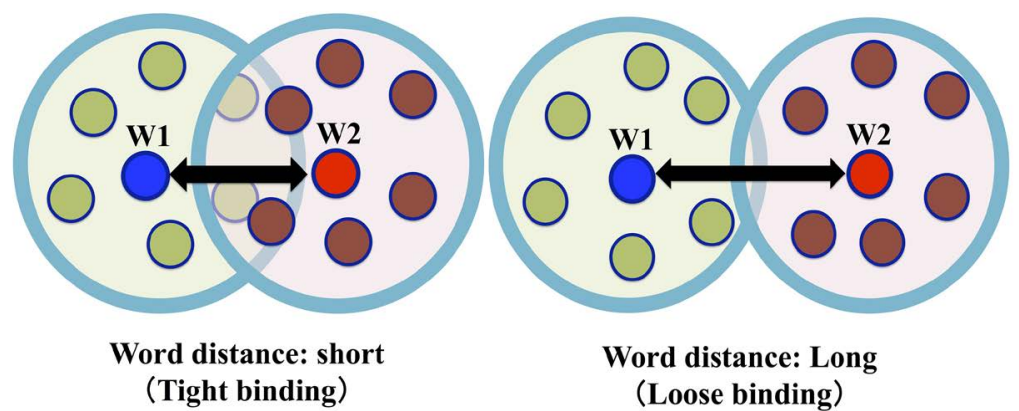

Figure 8. A word network model for relational memory formation between two words (W1 and W2). The words to be memorized are associated by parallel retrieval during encoding. In this model, these associates are allocated in proximity to each other, thereby constructing a word cluster. As semantic distance between two words decreases, between-cluster distance decreases, promoting relational memory formation. 
activities of the deep brain neural structures (e.g., dACC and upper brainstem) and conducted in a short time window of approximately $<1 \mathrm{~s}$. Such a short time window may not impede time-limited $(<2 s)$ enhancement of STP by dopaminergic neural activity in reward systems [68] and may further provide benefits of avoiding excitotoxicity [69]; however, future studies are required to provide biochemical evidence for this claim.

\subsection{Instruction Effects}

We further examined differences on the ER-DBA traces between related and unrelated pairs in encoding. dACC was deactivated during the presentation of the first word (W1) for related pairs while no deactivation was observed for unrelated pairs. Deactivation for the related condition cannot be explained by the model with parallel retrieval benefits (Figure 8) because the related condition is considered to impose light cognitive load compared to the unrelated condition. We propose that deactivation for related pairs is attributable to an instruction effect. According to a previous study on human learning [70], instruction effects constitute a strategy for engaging requirements for best effort while saving costs. In our study, the effect was attributed to proactive behaviors of the subjects to retrieve words associated with the first word (W1). Since subjects were instructed in advance about whether presented pairs were related or unrelated, such proactive retrieval was beneficial for related pairs. On the other hand, the strategy was inactivated for the unrelated condition because subjects knew that associates were unrelated to the second word and word retrieval would be futile. We suggest that such a strategy can also be attributed to economic decision-making as a function of dACC activation.

\subsection{Memory Dysfunction Mechanisms Predicted by Dynamics of dACC}

We elicited that the deactivation of dACC, associated with hyperpolarization for generating giant depolarizing potentials, was essential for encoding success in relational memory formation. According to our recent study [47], such dACC deactivation is correlated with the activity of the upper brainstem including the monoaminergic neural systems in the ventral tegmental areas. Hence, a possible factor of memory dysfunction can be the decline of monoaminergic neural activities in such deep-brain structures [71] [72].

Another factor is the age-related impairment [73] of GABAergic neural systems in dACC essential for hyperpolarization [74]. The impairment in such neural systems causes cognitive impairment [75], including amnesia in early stages, but continuous decline may modify the property of NMDA receptors as cytotoxic rather than protective [76].

For both cases, an insufficient deactivation of dACC is considered to be effective neurophysiological markers for detecting memory dysfunction in various diseases with memory dysfunction. 
To discuss memory dysfunction, we also have to mention excessive deactivation of dACC. As shown in Figure 7, strong deactivations degraded memory accuracy by increasing discordant responses with erroneously memorized words regarded as MA responses. This indicated that successful memory formation may be constructed by preventing unnecessary engrams during encoding and that excessive deactivation may limit such corrective functions. This claim is supported by some clinical experiences such as delusion in schizophrenia [77] and related pharmacological behavioral modulations using NMDAR antagonists accompanied with the compensation of dopaminergic system activations [78].

\subsection{Unresolved Issues}

The ER-DBA method is limited to dACC and reports no information on other brain areas, including the hippocampus and posterior cingulate cortex that are associated with the Papez circuit. These areas are thought to contribute to memory formation based on neural plasticity similar to that occurring in STP. However, relationships between dACC and other Papez-associated areas remain unclear. Future studies should conduct simultaneous EEG and fMRI measurements to explore the mechanisms underpinning relational memory formation.

This study was also limited to an electrophysiological investigation so direct measurement of NMDA effects using positron-emission tomography tracers [79] [80] is necessary to confirm our claims.

The findings of obtained in this study will contribute in eliciting neural mechanisms involved in memory impairment in various diseases typically including dementia, which has become a world-wide issue owing to its incredibly increasing prevalence in the last few decades [81]. However, this study was limited to investigations performed on healthy young subjects with no memory impairment. Future clinical studies with cooperation from high-risk patients with progression to Alzheimer's disease will further contribute to the findings of this study [82].

\section{Conclusion}

We investigated dynamic behaviors of dACC during word-pair tasks using a novel event-related deep brain activity (ER-DBA) method to uncover underlying mechanisms of relational memory formation. Our findings suggest that temporal deactivation of AACC is essential for successful encoding and recall of relational memory. Although retention from encoding until later recall was very short, initially encoded memories were robust, independent of intervention of other trials. This suggests that encoding was supported by short-term neural plasticity in a short time window of a few $100 \mathrm{~ms}$ provided by the deactivation dip. Such dACC dynamics in relational memory formation, which was detected for the first time by event-related deep brain activity method beyond the temporal limitation of conventional event-related fMRI methods, will be expected to not only contribute to eliciting whole mechanisms of durable memories but also 
provide novel neurophysiological markers for detecting memory dysfunctions.

\section{Acknowledgements}

This study was partially supported by JSPS KAKENHI Grant Number JP16K01307.

\section{References}

[1] Wagner, A.D., Schacter, D.L., Rotte, M., Koutstaal, W., Maril, A., Dale, A.M., Rosen, B.R. and Buckner, R.L. (1998) Building Memories: Remembering and Forgetting of Verbal Experiences as Predicted by Brain Activity. Science, 281, 1188-1191. https://doi.org/10.1126/science.281.5380.1188

[2] Daselaar, S.M., Prince, S.E. and Cabeza, R. (2004) When Less Means More: Deactivations during Encoding That Predict Subsequent Memory. Neuroimage, 23, 921-927. https://doi.org/10.1016/j.neuroimage.2004.07.031

[3] Prince, S.E., Daselaar, S.M. and Cabeza, R. (2005) Neural Correlates of Relational Memory: Successful Encoding and Retrieval of Semantic and Perceptual Associations. Journal of Neuroscience, 25, 1203-1210. https://doi.org/10.1523/JNEUROSCI.2540-04.2005

[4] Hannula, D.E., Tranel, D., Allen, J.S., Kirchhoff, B.A., Nickel, A.E. and Cohen, N.J. (2015) Memory for Items and Relationships among Items Embedded in Realistic Scenes: Disproportionate Relational Memory Impairments in Amnesia. Neuropsychology, 29, 126-138. https://doi.org/10.1037/neu0000119

[5] Daselaar, S.M., Prince, S.E., Dennis, N.A., Hayes, S.M., Kim, H. and Cabeza, R. (2009) Posterior Midline and Ventral Parietal Activity Is Associated with Retrieval Success and Encoding Failure. Frontiers in Human Neuroscience, 3, 13. https://doi.org/10.3389/neuro.09.013.2009

[6] Chai, X.J., Ofen, N., Gabrieli, J.D. and Whitfield-Gabrieli, S. (2014) Development of Deactivation of the Default-Mode Network during Episodic Memory Formation. Neuroimage, 84, 932-938. https://doi.org/10.1016/j.neuroimage.2013.09.032

[7] Huijbers, W., Pennartz, C.M., Cabeza, R. and Daselaar, S.M. (2011) The Hippocampus Is Coupled with the Default Network during Memory Retrieval But Not during Memory Encoding. PLoS ONE, 6, e17463.

https://doi.org/10.1371/journal.pone.0017463

[8] Wagner, I.C., van, Buuren, M., Bovy, L. and Fernández, G. (2016) Parallel Engagement of Regions Associated with Encoding and Later Retrieval Forms Durable Memories. Journal of Neuroscience, 36, 7985-7995. https://doi.org/10.1523/JNEUROSCI.0830-16.2016

[9] Kim, H. (2015) Encoding and Retrieval along the Long Axis of the Hippocampus and Their Relationships with Dorsal Attention and Default Mode Networks: The HERNET Model. Hippocampus, 25, 500-510. https://doi.org/10.1002/hipo.22387

[10] Oren, N., Shapira-Lichter, I., Lerner, Y., Tarrasch, R., Hendler, T., Giladi, N. and Ash, E.L. (2016) How Attention Modulates Encoding of Dynamic Stimuli. Frontiers in Human Neuroscience, 10, 507. https://doi.org/10.3389/fnhum.2016.00507

[11] Sneve, M.H., Grydeland, H., Nyberg, L., Bowles, B., Amlien, I.K., Langnes, E., Walhovd, K.B. and Fjell, A.M. (2015) Mechanisms Underlying Encoding of Short-Lived versus Durable Episodic Memories. Journal of Neuroscience, 35, 5202-5212. https://doi.org/10.1523/JNEUROSCI.4434-14.2015

[12] Rubin, R.D., Schwarb, H., Lucas, H.D., Dulas, M.R. and Cohen, N.J. (2017) Dynam- 
ic Hippocampal and Prefrontal Contributions to Memory Processes and Representations Blur the Boundaries of Traditional Cognitive Domains. Brain Sciences, 7, 82. https://doi.org/10.3390/brainsci7070082

[13] Babiloni, C., Vecchio, F., Rossi, S., De, Capua, A., Bartalini, S., Ulivelli, M. and Rossini, P.M. (2007) Human Ventral Parietal Cortex Plays a Functional Role on Visuospatial Attention and Primary Consciousness. A Repetitive Transcranial Magnetic Stimulation Study. Cerebral Cortex, 17, 1486-1492.

https://doi.org/10.1093/cercor/bhl060

[14] Cabeza, R., Ciaramelli, E. and Moscovitch, M. (2012) Cognitive Contributions of the Ventral Parietal Cortex: An Integrative Theoretical Account. Trends in Cognitive Science, 16, 338-352. https://doi.org/10.1016/j.tics.2012.04.008

[15] Daselaar, S.M., Huijbers, W., Eklund, K., Moscovitch, M. and Cabeza, R. (2013) Resting-State Functional Connectivity of Ventral Parietal Regions Associated with Attention Reorienting and Episodic Recollection. Frontiers Human Neuroscience, 7, 38. https://doi.org/10.3389/fnhum.2013.00038

[16] Bokde, A.L., Lopez-Bayo, P., Born, C., Ewers, M., Meindl, T., Teipel, S.J., Faltraco, F., Reiser, M.F., Möller, H.J. and Hampel, H. (2010) Alzheimer Disease: Functional Abnormalities in the Dorsal Visual Pathway. Radiology, 254, 219-226. https://doi.org/10.1148/radiol.2541090558

[17] Zhang, Z., Zheng, H., Liang, K., Wang, H., Kong, S., Hu, J., Wu, F. and Sun, G. (2015) Functional Degeneration in Dorsal and Ventral Attention Systems in Amnestic Mild Cognitive Impairment and Alzheimer's Disease: An fMRI Study. Neuroscience Letters, 585, 160-165. https://doi.org/10.1016/j.neulet.2014.11.050

[18] Franzmeier, N., Göttler, J., Grimmer, T., Drzezga, A., Áraque-Caballero, M.A., Simon-Vermot, L., Taylor, A.N.W., Bürger, K., Catak, C., Janowitz, D., Müller, C., Duering, M., Sorg, C. and Ewers, M. (2017) Resting-State Connectivity of the Left Frontal Cortex to the Default Mode and Dorsal Attention Network Supports Reserve in Mild Cognitive Impairment. Frontiers in Aging Neuroscience, 9, 264. https://doi.org/10.3389/fnagi.2017.00264

[19] Irish, M., Bunk, S., Tu, S., Kamminga, J., Hodges, J.R., Hornberger, M. and Piguet, O. (2015) Preservation of Episodic Memory in Semantic Dementia: The Importance of Regions beyond the Medial Temporal Lobes. Neuropsychologia, 81, 50-60. https://doi.org/10.1016/j.neuropsychologia.2015.12.005

[20] Tan, R.H., Wong, S., Kril, J.J., Piguet, O., Hornberger, M., Hodges, J.R. and Halliday, G.M. (2014) Beyond the Temporal Pole: Limbic Memory Circuit in the Semantic Variant of Primary Progressive Aphasia. Brain, 137, 2065-2076. https://doi.org/10.1093/brain/awu118

[21] Xie, C., Bai, F., Yu, H., Shi, Y., Yuan, Y., Chen, G., Li, W., Chen, G., Zhang, Z. and Li, S.J. (2012) Abnormal Insula Functional Network Is Associated with Episodic Memory Decline in Amnestic Mild Cognitive Impairment. Neuroimage, 63, 320-327. https://doi.org/10.1016/j.neuroimage.2012.06.062

[22] Zhuang, L., Sachdev, P.S., Trollor, J.N., Reppermund, S., Kochan, N.A., Brodaty, H. and Wen, W. (2013) Microstructural White Matter Changes, Not Hippocampal Atrophy, Detect Early Amnestic Mild Cognitive Impairment. PLoS ONE, 8, e58887. https://doi.org/10.1371/journal.pone.0058887

[23] Aggleton, J.P., Pralus, A., Nelson, A.J. and Hornberger, M. (2016) Thalamic Pathology and Memory Loss in Early Alzheimer's Disease: Moving the Focus from the Medial Temporal Lobe to Papez Circuit. Brain, 139, 1877-1890.

https://doi.org/10.1093/brain/aww083 
[24] Bergmann, H.C., Daselaar, S.M., Fernández, G. and Kessels, R.P. (2016) Neural Substrates of Successful Working Memory and Long-Term Memory Formation in a Relational Spatial Memory Task. Cognitive Processing, 17, 377-387. https://doi.org/10.1007/s10339-016-0772-7

[25] Wang, D.V. and Ikemoto, S. (2016) Coordinated Interaction between Hippocampal Sharp-Wave Ripples and Anterior Cingulate Unit Activity. Journal of Neuroscience, 36, 10663-10672. https://doi.org/10.1523/JNEUROSCI.1042-16.2016

[26] Tse, D., Takeuchi, T., Kakeyama, M., Kajii, Y., Okuno, H., Tohyama, C., Bito, H. and Morris, R.G. (2011) Schema-Dependent Gene Activation and Memory Encoding in Neocortex. Science, 333, 891-895. https://doi.org/10.1126/science.1205274

[27] Einarsson, E.Ö. and Nader, K. (2012) Involvement of the Anterior Cingulate Cortex in Formation, Consolidation, and Reconsolidation of Recent and Remote Contextual Fear Memory. Learning and Memmory, 19, 449-452.

https://doi.org/10.1101/lm.027227.112

[28] Weible, A.P., Rowland, D.C., Monaghan, C.K., Wolfgang, N.T. and Kentros, C.G. (2012) Neural Correlates of Long-Term Object Memory in the Mouse Anterior Cingulate Cortex. Journal of Neuroscience, 32, 5598-5608. https://doi.org/10.1523/JNEUROSCI.5265-11.2012

[29] Carter, C.S., Macdonald, A.M., Botvinick, M., Ross, L.L., Stenger, V.A., Noll, D. and Cohen, J.D. (2000) Parsing Executive Processes: Strategic vs. Evaluative Functions of the Anterior Cingulate Cortex. Proceedings of the National Academy of Sciences of the United States of America, 97, 1944-1948. https://doi.org/10.1073/pnas.97.4.1944

[30] Posner, M.I., Sheese, B.E., Odludaş, Y. and Tang, Y. (2006) Analyzing and Shaping Human Attentional Networks. Neural Networks, 19, 1422-1429. https://doi.org/10.1016/j.neunet.2006.08.004

[31] Sridharan, D., Levitin, D.J. and Menon, V. (2008) A Critical Role for the Right Fronto-Insular Cortex in Switching between Central-Executive and Default-Mode Networks. Proceedings of National Academy of Science of the United States of America, 105, 12569-12574. https://doi.org/10.1073/pnas.0800005105

[32] Seeley, W.W., Menon, V., Schatzberg, A.F., Keller, J., Glover, G.H., Kenna, H., Reiss, A.L. and Greicius, M.D. (2007) Dissociable Intrinsic Connectivity Networks for Salience Processing and Executive Control. Journal of Neuroscience, 27, 2349-2356. https://doi.org/10.1523/JNEUROSCI.5587-06.2007

[33] Menon, V. and Uddin, L.Q. (2010) Saliency, Switching, Attention and Control: A Network Model of Insula Function. Brain Structure and Function, 214, 655-667. https://doi.org/10.1007/s00429-010-0262-0

[34] Manza, P., Hu, S., Chao, H.H., Zhang, S., Leung, H.C. and Li, C.R. (2016) A Dual But Asymmetric Role of the Dorsal Anterior Cingulate Cortex in Response Inhibition and Switching from a Non-Salient to Salient Action. Neuroimage, 134, 466-474. https://doi.org/10.1016/j.neuroimage.2016.04.055

[35] Bohgaki, T., Katagiri, Y. and Usami, M. (2014) Pain-Relief Effects of Aroma Touch Therapy with Citrus Junos Oil Evaluated by Quantitative EEG Occipital Alpha-2 Rhythm Powers. Journal of Behavioral and Brain Science, 4, 43-48. https://doi.org/10.4236/jbbs.2014.41002

[36] Imai, E., Katagiri, Y., Hosaka, H. and Itao, K. (2016) Individual Differences in Cognitive Performance Regulated by Deep-Brain Activity during Mild Passive Hyperthermia and Neck Cooling. Journal of Behavioral and Brain Science, 6, 305-316. https://doi.org/10.4236/jbbs.2016.68030 
[37] Sadato, N., Nakamura, S., Oohashi, T., Nishina, E., Fuwamoto, Y., Waki, A. and Yonekura, Y. (1998) Neural Networks for Generation and Suppression of Alpha Rhythm: A PET Study. NeuroReport, 9, 893-897. https://doi.org/10.1097/00001756-199803300-00024

[38] Goldman, R.I., Stern, J.M., Engel, J. and Cohen, M.S. (2002) Simultaneous EEG and fMRI of the Alpha Rhythm. NeuroReport, 13, 2487-2492. https://doi.org/10.1097/00001756-200212200-00022

[39] Schreckenberger, M., Lange-Asschenfeldt, C., Lochmann, M., Mann, K., Siessmeier, T., Buchholz, H.G., Bartenstein, P. and Gründer, G. (2004) The Thalamus as the Generator and Modulator of EEG Alpha Rhythm: A Combined PET/EEG Study with Lorazepam Challenge in Humans. Neuroimage, 22, 637-644.

https://doi.org/10.1016/j.neuroimage.2004.01.047

[40] Omata, K., Hanakawa, T., Morimoto, M. and Honda, M. (2013) Spontaneous Slow Fluctuation of EEG Alpha Rhythm Reflects Activity in Deep-Brain Structures: A Simultaneous EEG-fMRI Study. PLoS ONE, 8, e66869.

https://doi.org/10.1371/journal.pone.0066869

[41] Liu, T.T. (2012) The Development of Event-Related fMRI Designs. Neuroimage, 62, 1157-1162. https://doi.org/10.1016/j.neuroimage.2011.10.008

[42] Ikkai, A., Blacker, K.J., Lakshmanan, B.M., Ewenm, J.B. and Courtney, S.M. (2014) Maintenance of Relational Information in Working Memory Leads to Suppression of the Sensory Cortex. Journal of Neurophysiolgy, 112, 1903-1915. https://doi.org/10.1152/jn.00134.2014

[43] van Geldorp, B., Parra, M.A. and Kessels, R.P. (2015) Cognitive and Neuropsychological Underpinnings of Relational and Conjunctive Working Memory Binding across Age. Memory, 23, 1112-1122. https://doi.org/10.1080/09658211.2014.953959

[44] Libby, L.A., Hannula, D.E. and Ranganath, C. (2014) Medial Temporal Lobe Coding of Item and Spatial Information during Relational Binding in Working Memory. Journal of Neuroscience, 34, 14233-14242. https://doi.org/10.1523/JNEUROSCI.0655-14.2014

[45] Ongür, D., Cullen, T.J., Wolf, D.H., Rohan, M., Barreira, P., Zalesak, M. and Heckers, S. (2006) The Neural Basis of Relational Memory Deficits in Schizophrenia. Archives of General Psychiatry, 63, 356-365. https://doi.org/10.1001/archpsyc.63.4.356

[46] Japan Society for Higher Brain Dysfunction (2014) Standard Verbal Paired-Associate Learning Test; S-PA. http://www.higherbrain.or.jp/main.html

[47] Imai, E. and Katagiri, Y. (2018) Cognitive Control and Brain Network Dynamics during Word Generation Tasks Predicted using a Novel Event-Related Deep Brain Activity Method. Journal of Behavioral and Brain Science, 8, 93-115. https://doi.org/10.4236/jbbs.2018.82006

[48] Yang, J.X., Hua, L., Li, Y.Q., Jiang, Y.Y., Han, D., Liu, H., Tang, Q.Q., Yang, X.N., Yin, C., Hao, L.Y., Yu, L., Wu, P., Shao, C.J., Ding, H.L., Zhang, Y.M. and Cao, J.L. (2015) Caveolin-1 in the Anterior Cingulate Cortex Modulates Chronic Neuropathic Pain via Regulation of NMDA Receptor 2B Subunit. Journal of Neuroscience, 35, 36-52. https://doi.org/10.1523/JNEUROSCI.1161-14.2015

[49] Holloway-Erickson, C.M., McReynolds, J.R. and McIntyre, C.K. (2012) Memory-Enhancing Intra-Basolateral Amygdala Infusions of Clenbuterol Increase Arc and CaMKII $\alpha$ Protein Expression in the Rostral Anterior Cingulate Cortex. Frontiers in Behavioral Neuroscience, 6, 17. https://doi.org/10.3389/fnbeh.2012.00017

[50] Song, Q., Zheng, H.W., Li, X.H., Huganir, R.L., Kuner, T., Zhuo, M. and Chen, T. 
(2017) Selective Phosphorylation of AMPA Receptor Contributes to the Network of Long-Term Potentiation in the Anterior Cingulate Cortex. Journal of Neuroscience, 37, 8534-8548. https://doi.org/10.1523/JNEUROSCI.0925-17.2017

[51] Koga, K., Yao, I., Setou, M. and Zhuo, M. (2017) SCRAPPER Selectively Contributes to Spontaneous Release and Presynaptic Long-Term Potentiation in the Anterior Cingulate Cortex. Journal of Neuroscience, 37, 3887-3895. https://doi.org/10.1523/JNEUROSCI.0023-16.2017

[52] Chen, T., Lu, J., Song, Q., Liu, M.G., Koga, K., Descalzi, G., Li, Y.Q. and Zhuo, M. (2014) Pharmacological Rescue of Cortical Synaptic and Network Potentiation in a Mouse Model for Fragile X Syndrome. Neuropsychopharmacology, 39, 1955-1967. https://doi.org/10.1038/npp.2014.44

[53] Zhuo, M. (2013) Long-Term Potentiation in the Anterior Cingulate Cortex and Chronic Pain. Philosophical Transactions of the Royal Society of London. Series B, Biological Sciences, 369, Article ID: 20130146. https://doi.org/10.1098/rstb.2013.0146

[54] Chen, T., O’Den, G., Song, Q., Koga, K., Zhang, M.M. and Zhuo, M. (2014) Adenylyl Cyclase Subtype 1 Is Essential for Late-Phase Long Term Potentiation and Spatial Propagation of Synaptic Responses in the Anterior Cingulate Cortex of Adult Mice. Molecular Pain, 10, 65. https://doi.org/10.1186/1744-8069-10-65

[55] Frankland, P.W., Bontempi, B., Talton, L.E., Kaczmarek, L. and Silva, A.J. (2004) The Involvement of the Anterior Cingulate Cortex in Remote Contextual Fear Memory. Science, 304, 881-883. https://doi.org/10.1126/science.1094804

[56] Lee, C.M., Sylantyev, S. and Shyu, B.C. (2006) Short-Term Synaptic Plasticity in Layer II/III of the Rat Anterior Cingulate Cortex. Brain Research Bulletin, 71, 416-427. https://doi.org/10.1016/j.brainresbull.2006.10.011

[57] Gemmell, C. and O'Mara, S.M. (2002) Plasticity in the Projection from the Anterior Thalamic Nuclei to the Anterior Cingulate Cortex in the Rat in Vivo: Paired-Pulse Facilitation, Long-Term Potentiation and Short-Term Depression. Neuroscience, 109, 401-406. https://doi.org/10.1016/S0306-4522(01)00554-1

[58] Castro-Alamancos, M.A. and Connors, B.W. (1996) Cellular Mechanisms of the Augmenting Response: Short-Term Plasticity in a Thalamocortical Pathway. Journal of Neuroscience, 16, 7742-7756. https://doi.org/10.1523/JNEUROSCI.16-23-07742.1996

[59] Khalilov, I., Minlebaev, M., Mukhtarov, M. and Khazipov, R. (2015) Dynamic Changes from Depolarizing to Hyperpolarizing GABAergic Actions during Giant Depolarizing Potentials in the Neonatal Rat Hippocampus. Journal of Neuroscience, 35, 12635-12642. https://doi.org/10.1523/JNEUROSCI.1922-15.2015

[60] McQuail, J.A., Beas, B.S., Kelly, K.B., Simpson, K.L., Frazier, C.J., Setlow, B. and Bizon, J.L. (2016) NR2A-Containing NMDARs in the Prefrontal Cortex Are Required for Working Memory and Associated with Age-Related Cognitive Decline. Journal of Neuroscience, 36, 12537-12548. https://doi.org/10.1523/JNEUROSCI.2332-16.2016

[61] Duarte, A., Ranganath, C., Winward, L., Hayward, D. and Knight, R.T. (2004) Dissociable Neural Correlates for Familiarity and Recollection during the Encoding and Retrieval of Pictures. Cognitive Brain Research, 18, 255-272.

https://doi.org/10.1016/j.cogbrainres.2003.10.010

[62] Hoang, T.H., Aliane, V. and Manahan-Vaughan, D. (2018) Novel Encoding and Updating of Positional, or Directional, Spatial Cues Are Processed by Distinct Hippocampal Subfields: Evidence for Parallel Information Processing and the "What" 
Stream. Hippocampus, 28, 315-326. https://doi.org/10.1002/hipo.22833

[63] You, Y. and Li, W. (2016) Parallel Processing of General and Specific Threat during Early Stages of Perception. Social Cognitive Affective Neuroscience, 11, 395-404. https://doi.org/10.1093/scan/nsv123

[64] Abdel, Rahman, R. and Sommer, W. (2003) Does Phonological Encoding in Speech Production Always Follow the Retrieval of Semantic Knowledge? Electrophysiological Evidence for Parallel Processing. Cognitive Brain Research, 16, 372-382. https://doi.org/10.1016/S0926-6410(02)00305-1

[65] Bruffaerts, R., Dupont, P., Peeters, R., De Deyne, S., Storms, G. and Vandenberghe, R. (2013) Similarity of fMRI Activity Patterns in Left Perirhinal Cortex Reflects Semantic Similarity between Words. Journal of Neuroscience, 33, 18597-18607. https://doi.org/10.1523/JNEUROSCI.1548-13.2013

[66] Meyniel, F., Safra, L. and Pessiglione, M. (2014) How the Brain Decides When to Work and When to Rest: Dissociation of Implicit-Reactive from Explicit-Predictive Computational Processes. PLOS Computational Biology, 10, e1003584. https://doi.org/10.1371/journal.pcbi.1003584

[67] Farrell, A.M., Goh, J.O.S. and White, B.J. (2018) Financial Incentives Differentially Regulate Neural Processing of Positive and Negative Emotions during Value-Based Decision-Making. Frontiers in Human Neuroscience, 12, 58. https://doi.org/10.3389/fnhum.2018.00058

[68] Yagishita, S., Hayashi-Takagi, A., Ellis-Davies, G.C., Urakubo, H., Ishii, S. and Kasai, H. (2014) A Critical Time Window for Dopamine Actions on the Structural Plasticity of Dendritic Spines. Science, 345, 1616-1620. https://doi.org/10.1126/science.1255514

[69] Zhou, X., Hollern, D., Liao, J., Andrechek, E. and Wang, H. (2013) NMDA Receptor-Mediated Excitotoxicity Depends on the Coactivation of Synaptic and Extrasynaptic Receptors. Cell Death and Disease, 4, e560. https://doi.org/10.1038/cddis.2013.82

[70] Navarro, A., Arriola, N. and Alonso, G. (2016) Instruction-Driven Processing in Human Perceptual Learning. The Quarterly Journal of Experimental Psychology, 69, 1583-1605. https://doi.org/10.1080/17470218.2015.1088556

[71] Trillo, L., Das, D., Hsieh, W., Medina, B., Moghadam, S., Lin, B., Dang, V., Sanchez, M.M., De, Miguel, Z., Ashford, J.W. and Salehi, A. (2013) Ascending Monoaminergic Systems Alterations in Alzheimer's Disease. Translating Basic Science into Clinical Care. Neuroscience and Biobehavioral Reviews, 37, 1363-1379. https://doi.org/10.1016/j.neubiorev.2013.05.008

[72] Kushida, S., Kimoto, K., Hori, N., Toyoda, M., Karasawa, N., Yamamoto, T., Kojo, A. and Onozuka, M. (2008) Soft-Diet Feeding Decreases Dopamine Release and Impairs Aversion Learning in Alzheimer Model Rats. Neuroscience Letters, 439, 208-211. https://doi.org/10.1016/j.neulet.2008.05.017

[73] Rozycka, A. and Liguz-Lecznar, M. (2017) The Space Where Aging Acts: Focus on the GABAergic Synapse. Aging Cell, 16, 634-643. https://doi.org/10.1111/acel.12605

[74] Czarnecki, A., Le, Corronc, H., Rigato, C., Le, Bras, B., Couraud, F., Scain, A.L., Allain, A.E., Mouffle, C., Bullier, E., Mangin, J.M., Branchereau, P. and Legendre, P. (2014) Acetylcholine Controls GABA-, Glutamate-, and Glycine-Dependent Giant Depolarizing Potentials that Govern Spontaneous Motoneuron Activity at the Onset of Synaptogenesis in the Mouse Embryonic Spinal Cord. The Journal of Neuroscience, 34, 6389-6404. https://doi.org/10.1523/JNEUROSCI.2664-13.2014

[75] Porges, E.C., Woods, A.J., Edden, R.A., Puts, N.A., Harris, A.D., Chen, H., Garcia, 
A.M., Seider, T.R., Lamb, D.G., Williamson, J.B. and Cohen, R.A. (2016) Frontal Gamma-Aminobutyric Acid Concentrations Are Associated with Cognitive Performance in Older Adults. Biological Psychiatry. Cognitive Neuroscience and Neuroimaging, 2, 38-44.

[76] Marczynski, T.J. (1998) GABAergic Deafferentation Hypothesis of Brain Aging and Alzheimer's Disease Revisited. Brain Research Bulletin, 45, 341-379. https://doi.org/10.1016/S0361-9230(97)00347-X

[77] Liu, Y.C., Tang, C.C., Hung, T.T., Tsai, P.C. and Lin, M.F. (2018) The Efficacy of Metacognitive Training for Delusions in Patients with Schizophrenia: A Meta-Analysis of Randomized Controlled Trials Informs Evidence-Based Practice. Worldviews on Evidence-Based Nursing, 15, 130-139.

https://doi.org/10.1111/wvn.12282

[78] Gottlieb, J.D., Cather, C., Shanahan, M., Creedon, T., Macklin, E.A. and Goff, D.C. (2011) D-Cycloserine Facilitation of Cognitive Behavioral Therapy for Delusions in Schizophrenia. Schizophrenia Research, 131, 69-74. https://doi.org/10.1016/j.schres.2011.05.029

[79] McGinnity, C.J., Hammers, A., Riaño, Barros, D.A., Luthra, S.K., Jones, P.A., Trigg, W., Micallef, C., Symms, M.R., Brooks, D.J., Koepp, M.J. and Duncan, J.S. (2014) Initial Evaluation of 18F-GE-179, a Putative PET Tracer for Activated N-methyl D-aspartate Receptors. Journal of Nuclear Medicine, 55, 423-430. https://doi.org/10.2967/jnumed.113.130641

[80] Klein, P.J., Schuit, R.C., Metaxas, A., Christiaans, J.A.M., Kooijman, E., Lammertsma, A.A., van, Berckel, B.N.M. and Windhorst, A.D. (2017) Synthesis, Radiolabeling and Preclinical Evaluation of a [11C]GMOM Derivative as PET Radiotracer for the Ion Channel of the N-methyl-D-aspartate Receptor. Nuclear Medicine and Biology, 51, 25-32. https://doi.org/10.1016/j.nucmedbio.2017.05.003

[81] Prince, M., Bryce, R., Albanese, E., Wimo, A., Ribeiro, W. and Ferri, C.P. (2013) The Global Prevalence of Dementia: A Systematic Review and Metaanalysis. Alzheimer's and Dementia: The Journal of the Alzheimer's Association, 9, 63-75. https://doi.org/10.1016/j.jalz.2012.11.007

[82] Quaranta, D., Gainotti, G., Di, Giuda, D., Vita, M.G., Cocciolillo, F., Lacidogna, G., Guglielmi, V., Masullo, C., Giordano, A. and Marra, C. (2018) Predicting Progression of Amnesic MCI: The Integration of Episodic Memory Impairment with Perfusion SPECT. Psychiatry Research: Neuroimaging, 271, 43-49.

https://doi.org/10.1016/j.pscychresns.2017.10.008 